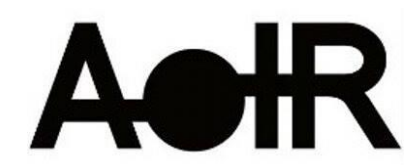

Selected Papers of \#AoIR2021:

The 22nd Annual Conference of the

Association of Internet Researchers

Virtual Event / 13-16 Oct 2021

\title{
THE QUALITY OF SURVEY SCALES FOR MEASURING INFORMATION PRIVACY CONCERNS ON SOCIAL NETWORK SITES: A SYSTEMATIC REVIEW
}

Jošt Bartol

University of Ljubljana

Andraž Petrovčič

University of Ljubljana

Vasja Vehovar

University of Ljubljana

\section{Introduction}

Information privacy concerns (IPCs) play an important role in user behavior on social network sites (SNSs). In general, IPCs are beliefs of individuals or groups regarding the possible collection and abuse of their personal information. These concerns are important in SNS contexts because users with higher IPCs are less likely to attain certain outcomes, arguably the most important of which is building social capital (Ellison et al., 2011). Moreover, information privacy is increasingly relevant to digital divide research given that similar sociodemographic factors have been found to influence internet skills, online privacy literacy, and IPCs (Epstein \& Quinn, 2020). Privacy is the key for individuals to maintain their "independence, freedom, and economic and social well-being" (Epstein \& Quinn, 2020, p. 8), and is therefore receiving increased attention among internet researchers.

Information privacy research has a long tradition, and the field is characterized by a range of disparate conceptual frameworks (Yun et al., 2019). This divergence resonates in the lack of a unified approach to assessing IPCs in particular because although many survey scales have been developed to measure these concerns and test their relationships to other internet phenomena, evidence of their validity has been scarce and inconsistent (Bélanger \& Crossler, 2011). Moreover, researchers have often adapted existing IPC scales to specific online contexts without following appropriate procedures (Preibusch, 2013). All this can affect generalizability and therefore prevent direct comparisons between studies.

Suggested Citation (APA): Bartol, J., Petrovčič, A., \& Vehovar, V. (2021, October). The quality of survey scales for measuring information privacy concerns on social network sites: A systematic review. Paper presented at AolR 2021: The 22nd Annual Conference of the Association of Internet Researchers. Virtual Event: AolR. Retrieved from http://spir.aoir.org. 
Accordingly, the aims of this research were to systematically examine the quality of survey scales used for measuring IPCs among SNS users and to provide suggestions for improving IPC measurement in the future. Thus, we addressed the following research questions: (RQ1) Which survey scales were used to measure the IPCs of SNS users? (RQ2) Which dimensions do these survey scales include? (RQ3) What are their psychometric properties?

\section{Method}

This study applied the COnsensus-based Standards for the selection of health Measurement INstruments (COSMIN) methodology for conducting systematic reviews of self-reported survey scales (Prinsen et al., 2018). The PRISMA-S guidelines were used for conducting, structuring, and presenting the literature search (Moher et al., 2009).

The search was conducted in June 2020 and covered 127 information sources, including Scopus, Web of Science, EBSCO, and PsycINFO. The search focused on articles published prior to January 1, 2020 that (1) focused on IPCs in the SNS context; (2) used a sample that consisted exclusively of SNS users; and (3) implemented an existing IPC survey scale or followed at least minimal standards in developing a new scale. Only peer-reviewed articles, conference proceedings papers, books, and book chapters in English were considered eligible.

After identifying the eligible articles, we extracted the source, length, dimensionality, and response options of each scale, and then evaluated its psychometric properties according to the COSMIN methodology. The assessed properties were structural validity, internal consistency, construct validity, measurement invariance, and criterion validity.

\section{Results}

The database searches retrieved a total of 2,606 articles. After removing the 1,181 duplicates, the remaining 1,425 abstracts were screened and, of these, 362 were retained for full-text assessment. Ultimately, 71 papers were deemed eligible for further analysis and included 35 different IPC survey scales.

The most frequently used IPC survey scale ( 22 articles) is the scale by Dinev and Hart (2006). Their scale contains four items that focus on concerns about what others might do with an individual's personal information found online. The second most frequently used scale is the Concern for Information Privacy (CFIP) instrument developed by Smith et al. (1996) which appears in five articles. The CFIP consists of 15 items that measure four distinct dimensions, namely collection, errors, unauthorized secondary use, and unauthorized access. The remaining 33 scales are used in four or fewer articles.

When further analyzing dimensionality, we found that 10 of the 35 IPC survey scales are multidimensional. Of the 18 dimensions identified, access, collection, and control are present in six different survey scales, followed by unauthorized secondary use (four 
scales), awareness (three), and errors (three). Each of the other 12 dimensions appeared only once. The results regarding the psychometric properties indicate that many of the scales are of questionable quality. Structural validity is particularly problematic. While it was assessed in most studies, the methods were often lacking. Moreover, although the scales were used in various countries, only four of them were tested for measurement invariance. These findings point to the need for more rigorous testing of structural validity and also for validation of IPC scales across different SNS populations and in different cultures.

\section{Conclusion}

The results confirm previous findings about the multitude of survey measures for assessing IPCs online (Preibusch, 2013). Despite the prevalence of unidimensional scales, the dimensions that seem essential in capturing the main aspects of IPCs in the SNS context are access, collection, and control. However, our results suggest that many IPC survey scales are of questionable quality. Nonetheless, we also identified some scales that are promising candidates for future use (e.g., Smith et al., 1996; Dinev \& Hart, 2006), although further testing and potential improvements are needed. In addition, the present study can provide the basis for a possible unified approach to survey measurement of IPCs in SNS contexts. Such an approach would enable direct comparisons between studies, accumulation of knowledge, and, consequently, the creation of a comprehensive conceptual framework for understanding and comparing the roles and implications of IPCs across various SNS platforms.

\section{References}

Bélanger, F., \& Crossler, R. E. (2011). Privacy in the digital age: A review of information privacy research in information systems. MIS Quarterly, 35(4), 1017-1041. https://doi.org/10.2307/41409971

Dinev, T., \& Hart, P. (2006). An extended privacy calculus model for e-commerce transactions. Information Systems Research, 17(1), 61-80. https://doi.org/10.1287/isre.1060.0080

Ellison, N. B., Vitak, J., Steinfield, C., Gray, R., \& Lampe, C. (2011). Negotiating privacy concerns and social capital needs in a social media environment. In S. Trepte \& L. Reinecke (Eds.), Privacy Online: Perspectives on Privacy and Self-Disclosure in the Social Web (pp. 19-32). Springer Berlin Heidelberg. https://doi.org/10.1007/978-3-642-21521-6_3

Epstein, D., \& Quinn, K. (2020). Markers of online privacy marginalization: Empirical examination of socioeconomic disparities in social media privacy attitudes, literacy, and behavior. Social Media and Society, 6(2). https://doi.org/10.1177/2056305120916853

Moher, D., Liberati, A., Tetzlaff, J., \& Altman, D. G. (2009). Preferred reporting items for systematic reviews and meta-analyses: The PRISMA statement. BMJ (Online), 339(7716), 332-336. https://doi.org/10.1136/bmj.b2535 
Preibusch, S. (2013). Guide to measuring privacy concern: Review of survey and observational instruments. International Journal of Human Computer Studies, 71(12), 1133-1143. https://doi.org/10.1016/j.ijhcs.2013.09.002

Prinsen, C. A. C., Mokkink, L. B., Bouter, L. M., Alonso, J., Patrick, D. L., de Vet, H. C. W., \& Terwee, C. B. (2018). COSMIN guideline for systematic reviews of patientreported outcome measures. Quality of Life Research, 27(5), 1147-1157. https://doi.org/10.1007/s11136-018-1798-3

Smith, J. H., Milberg, S. J., \& Burke, S. J. (1996). Information privacy: Measuring individuals' concerns about organizational practices. MIS Quarterly, 20(2), 167196. https://doi.org/10.2307/249477

Yun, H., Lee, G., \& Kim, D. J. (2019). A chronological review of empirical research on personal information privacy concerns: An analysis of contexts and research constructs. Information and Management, 56(4), 570-601.

https://doi.org/10.1016/j.im.2018.10.001 